\title{
Air-Borne and Tissue-Borne Sensitivities of Bioacoustic Sensors Used on the Skin Surface
}

\author{
Matías Zañartu*, Student Member, IEEE, Julio C. Ho, Student Member, IEEE, \\ Steve S. Kraman, Senior Member, IEEE, Hans Pasterkamp, Jessica E. Huber, \\ and George R. Wodicka, Fellow, IEEE
}

\begin{abstract}
Measurements of body sounds on the skin surface have been widely used in the medical field and continue to be a topic of current research, ranging from the diagnosis of respiratory and cardiovascular diseases to the monitoring of voice dosimetry. These measurements are typically made using light-weight accelerometers and/or air-coupled microphones attached to the skin. Although normally neglected, air-borne sounds generated by the subject or other sources of background noise can easily corrupt such recordings, which is particularly critical in the recording of voiced sounds on the skin surface. In this study, the sensitivity of commonly used bioacoustic sensors to air-borne sounds was evaluated and compared with their sensitivity to tissue-borne body sounds. To delineate the sensitivity to each pathway, the sensors were first tested in vitro and then on human subjects. The results indicated that, in general, the air-borne sensitivity is sufficiently high to significantly corrupt body sound signals. In addition, the air-borne and tissue-borne sensitivities can be used to discriminate between these components. Although the study is focused on the evaluation of voiced sounds on the skin surface, an extension of the proposed methods to other bioacoustic applications is discussed.
\end{abstract}

Index Terms-Accelerometers, acoustic transducers, biomedical acoustics, biomedical transducers, microphones, respiratory acoustics, skin vibration, speech analysis.

\section{INTRODUCTION}

$\mathbf{T}$ HE assessment of sound on the skin surface, better known as auscultation, provides a noninvasive approach to examining the circulatory, respiratory, gastrointestinal, and phonatory systems. The bioacoustic transducers used during recordings for this purpose are generally light-weight accelerometers and/or air-coupled microphones attached to the skin. Although intended to capture tissue-borne sounds, the sensors can also

Manuscript received May 13, 2008. First published October 31, 2008; current version published March 25, 2009. Asterisk indicates corresponding author

*M. Zañartu is with the School of Electrical and Computer Engineering, Purdue University, West Lafayette, IN 47907 USA (e-mail: mzanartu@ purdue. edu).

J. C. Ho is with the Weldon School of Biomedical Engineering, Purdue University, West Lafayette, IN 47907 USA (e-mail: hoj@purdue.edu).

S. S. Kraman is with the Department of Internal Medicine, University of Kentucky, Lexington, KY 40536 USA (e-mail: sskram01@email.uky.edu).

H. Pasterkamp is with the Department of Pediatrics and Child Health, University of Manitoba, Winnipeg, MB R3A 1S1, Canada (e-mail: pasterk@ cc.umanitoba.ca)

J. E. Huber is with the Department of Speech, Language, and Hearing Sciences, Purdue University, West Lafayette, IN 47907 USA (e-mail: jhuber@ purdue.edu).

G. R. Wodicka is with the Weldon School of Biomedical Engineering and the School of Electrical and Computer Engineering, Purdue University, West Lafayette, IN 47907 USA (e-mail: wodicka@ purdue.edu).

Digital Object Identifier 10.1109/TBME.2008.2008165 detect air-borne transmitted sounds generated by the subject (such as speech) and other sources of background noise. This corrupting signal is generally not taken into account in skin surface measurements under the assumption that it is negligible. The sensitivity to air-borne sounds is rarely provided in the manufacturer specifications, and if reported, such information often cannot be related directly to the actual performance of the sensor when it is mounted on a compliant surface such as human skin. Although there are some guidelines and standards to evaluate the air-borne and structure-borne sensitivity of sensors [1], [2], these guidelines are neither intended nor fully appropriate for biomedical acoustics applications. Even though there have been efforts to compare the tissue-borne sensitivity of commonly used bioacoustic sensors [3]-[6] and standardize these measurements for skin applications [7], [8], the air-borne sensitivity of bioacoustic sensors and its relation with the tissueborne sensitivity has not been consistently investigated.

During recordings of lung and heart sounds, typical sources of background noise that are transmitted via air-borne pathway are the subject's respiration and room noise. These components tend to be sufficiently uncorrelated with the biomedical signals of interest, the latter of which can be retrieved by means of signal processing techniques [9], [10]. In addition, it is common in clinical practice to make these recordings under relatively low levels of background noise, thus minimizing its influence during the recordings. However, skin surface recordings that require the subject to speak impose a larger challenge. Under this scenario, the subject's radiated voice in the room will generate a larger background noise that can be highly correlated with the skin vibration, making discrimination between air-borne and tissue-borne components difficult to achieve. These types of recordings of voiced sounds at the skin surface have been used, for instance, to detect respiratory anomalies by means of pectoriloquy or egophony [11]-[15], evaluate voice dosimetry [16]-[18], monitor singing techniques [19], predict sound pressure level (SPL) of voiced sounds [20], and evaluate nasalization [21]-[23], among others. In all of these studies, little or no attention has been paid to the effect of corruption in the tissue-borne signal due to undesired air-borne components. The adverse effect of air-borne sound may hamper observations that affect clinical monitoring and diagnosis of vocal and respiratory pathologies. The corrupting effect introduced by airborne transmitted components may help to explain some discrepancies and uncertainties observed in these and other related studies [24], [25]. Therefore, understanding the relative sensitivity of bioacoustic sensors to the tissue-borne and air-borne 
components becomes a critical point in order to formulate accurate claims based on skin surface measurements.

An ideal sensor would have a high tissue-borne sensitivity and low (or null) response to air-borne transmitted sounds. Actual bioacoustic sensors have frequency-dependent sensitivities and limited bandwidths of operation, making the criteria to discriminate between air-borne and tissue-borne components more challenging. Defining procedures to measure the sensitivities for each component and introducing simple guidelines to use them to discriminate between them are the primary goals of this study. Although the study is primarily focused on the evaluation of skin surface measurements of voiced sounds, many of the approaches and findings can be extrapolated to other applications such as lung and heart sounds measurements.

\section{MATERIALS AND METHODS}

The sensitivity of three commonly used bioacoustic sensors to air-borne sounds was evaluated and compared with their sensitivity to tissue-borne body sounds. The selected sensors were an air-coupled microphone (Sony ECM-77B), a lightweight accelerometer (Siemens EMT25C), and a very lightweight accelerometer (Knowles BU-7135). Herein, these sensors will be referred to as air-coupled microphone, Siemens, and Knowles. The sensors were amplified with a fixed gain using a Mackie mixer 1604-VLZ for the air-coupled microphone and two custom-made preamplifiers based on [5] and [17] for the accelerometers. The total sensors weight including cable and coating was $6.3 \mathrm{~g}$ for the air-coupled microphone, $15.8 \mathrm{~g}$ for the Siemens, and $0.8 \mathrm{~g}$ for the Knowles. These sensors have been used in a large number of applications related to the skin surface measurements of lung, cardiac, and voiced sounds (see, for example, [8], [16], [20], and [26]). Additional sensors such as a Polytec laser vibrometer (OFV3000 and OFV511), a B\&K hand-held analyzer (Type 2250), a B\&K microphone (Type 4191), and a PCB accelerometer (A353B17 and 483BO8) were used as reference sensors at different points in this study. Two different testing schemes were required to evaluate the air-borne and tissue-borne sensitivities separately. The methods and materials required for each scheme are described in the following sections.

\section{A. Tissue-Borne Sensitivity Experiments}

The procedure to evaluate the tissue-borne sensitivity of the sensors was achieved based on previous studies [7], [8]. The notion of a bioacoustic transducer tester (BATT) was further explored and used to quantify tissue-borne sensitivity. A BATT is an acoustic enclosure that allows only radiation of sound through a compliant artificial compound (Akton of $1 / 4$ in) that resembles human skin. The original BATT (herein referred to as BATT 1.0) was contrasted with two larger platforms (referred to as BATT 2.0 and 2.1). These new BATT designs are scaled versions (2:1 and 4:1, respectively) of the original BATT 1.0, with larger inner loudspeakers ( 3 and 6 in of diameter, respectively). For further details on the BATT design, see [7] and [8]. Larger testing platforms were observed to be better suited to excite low frequencies and allowed the effect of coverings that surround the sensor (e.g., a passive ear protector covering an air-coupled microphone) to be evaluated. Note that for the amplitude of vibration used in this study, the BATTs can also radiate airborne sound. However, the radiated sound was found to be at least $25 \mathrm{~dB}(\mathrm{~A})$ below the level estimated for voiced sounds, and thus, it was not expected to affect or corrupt the tissue-borne measurements.

The BATTs were excited with both voiced sounds and broadband pink noise, while the sensors were attached to the test surface of the BATTs using double-sided tape (3M 2181). An amplifier (Denon PMA-920) was used to drive the BATT loudspeaker. A prewhitening scheme was used to compensate for the overall transfer function of each BATT. This scheme is based on established principles [2], assuring that the BATT test surface vibrates with a flat response (in a logarithmic scale since it is based on pink noise) between $80 \mathrm{~Hz}$ and $8 \mathrm{kHz}$ with a maximum $2 \mathrm{~dB}$ deviation between neighboring one-third octave bands. This approach was convenient since it was set to match the criteria used later to calibrate the frequency response of airborne components. The prewhitening was performed using pink noise and a one-third octave equalizer (Behringer DEQ2496) and a reference sensor located on the center of the test surface. The manufacturer specifications for transverse shock sensitivity of very light-weight sensors are not expected to vary when placed on a more compliant surface such as that of the BATTs ( $1 / 4$ in of Akton, see [7] and [8] for more details). Based on this principle and given its miniature size $(\sim 6 \times 8 \times 2 \mathrm{~mm})$, very light weight, and flat and extended frequency response $(2 \mathrm{~Hz}-$ $6 \mathrm{kHz}$ within $5 \mathrm{~dB}$ ), the Knowles accelerometer was selected as a reference sensor for this calibration. It will be shown later in this paper that this sensor was also the least sensitive to air-borne sounds, which reaffirms its selection as a reference sensor in this case. The amplitude of vibration of the BATT test platforms was adjusted to resemble as closely as possible that of the skin vibration at the sternal notch (also known as jugular notch) during normal speech production of sustained vowels. This amplitude calibration was performed using the reference sensor after the aforementioned pre-whitening scheme.

All signals were filtered with an eight pole Butterworth lowpass filter (Krohn-Hite 3384) and digitized using a 16-bit data acquisition system (NI BNC-210) with a 96-kHz sampling frequency. Data post processing was performed using one-third octave band analysis according to [27]. Note that the logarithmic nature of this representation allows for a more clear appreciation of low-frequency phenomena.

All measurements were performed inside a soundproof chamber (IAC 102871). The background noise was measured according to [28] using a B\&K hand-held analyzer (Type 2250) obtaining an equivalent SPL (Leq Slow) of $20.9 \mathrm{~dB}(\mathrm{~A})$ or equivalently $74.1 \mathrm{~dB}(\mathrm{Z})$ (the $\mathrm{Z}$ notation incorporates no weighting factor between $12 \mathrm{~Hz}$ and $20 \mathrm{kHz}$ ).

\section{B. Air-Borne Sensitivity Experiments}

The air-borne sensitivity of bioacoustic sensors is a function of the material properties of the surface where they are attached. 


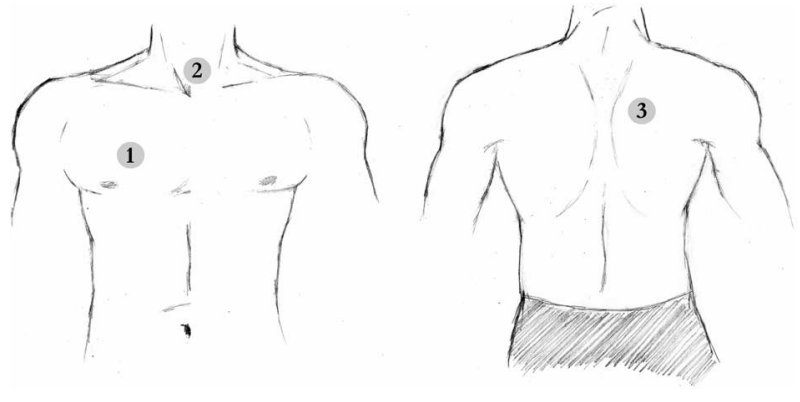

Fig. 1. Location of the sensors for the air-borne sensitivity tests in human subjects. (1) Second intercostal space over the anterior RUL of the lung. (2) Suprasternal notch. (3) Posterior RUL of the lung.

For the case of sensors placed on human skin, the body structure and skin compliance will create a combination that is not easily reproducible by artificial skin and mounting conditions. Even though certain artificial compounds (e.g., $1 / 4$ in of Akton) have been shown to have an acoustically comparable behavior with that of human skin [7], [8], the human body will provide a large and unique mounting configuration that is not well represented by the compounds themselves. Such mounting configuration is critical during the evaluation of the sensor's air-borne sensitivity since it dictates the extent of acoustic energy that is conveyed into transverse vibration of the test bed. Some artificial configurations can facilitate this transmission, thus distorting the airborne sensitivity experiments. This phenomenon was evident in preliminary in vitro tests, illustrating the difficulties associated with the design of artificial conditions that accurately mimic the skin/body mounting conditions for the sensors. The differences between artificial and human skin mounting conditions on the air-borne sensitivity are discussed in Section III-C.

On the other hand, the variability given by different human subjects is expected to be small. This assumption is given by the fact that during the recordings, each subject still remains in a controlled position without uttering speech and holding his/her breath at functional residual capacity. Therefore, factors such as height, weight — thus, body mass index (BMI) — and body structure are expected to have a minor effect on the local mounting conditions of the sensor. Given the low expected variations across subjects, the air-borne sensitivity of the bioacoustic sensor was tested using five human subjects. The actual subject variability is discussed in Section III-C. A relatively large set of measurements was first performed using one healthy young male reference subject, and a selected set of tests were performed using the additional subjects (two healthy young males and two healthy young females). The BMI of the subjects was observed between 23.2 and 26.2. The sensors were attached to the skin surfaces using double-sided tape (3M 2181) on three selected locations on the subject's body, as illustrated in Fig. 1. These locations are in accordance with the aforementioned applications of skin surface measurements.

The protocol used during these measurements is based on the principles established in [1] and [29], where a loudspeaker (Mackie HR 824) was placed 26 in from the anterior chest to achieve high levels of acoustic pressure $(60 \mathrm{~dB}$ over the noise floor). The background noise conditions were the same as previously described, for which an equivalent SPL of $100 \mathrm{~dB}(\mathrm{~A})$, or equivalently $105 \mathrm{~dB}(\mathrm{Z})$, was used. Although this level provides sufficient SNR to minimize the effects of background noise, it is not associated with normal speech levels. Measurements of SPLs during speech production are discussed in detail in [18] and [20]. These studies showed that typical SPL measurements at $30 \mathrm{~cm}$ from the mouth are generally $65 \mathrm{~dB}(Z)$ for soft voice, $75 \mathrm{~dB}(Z)$ for normal voice, and $85 \mathrm{~dB}(Z)$ for loud voice, all with an approximate $\pm 5 \mathrm{~dB}$ deviation for most of the cases. Observations made in our laboratory suggest that the SPL measured at $30 \mathrm{~cm}$ from the mouth compared with near-skin locations is $\sim 1 \mathrm{~dB}(Z)$ lower for the sternal notch, $\sim 3 \mathrm{~dB}(\mathrm{Z})$ lower for the anterior right upper lobe (RUL), and $\sim 12 \mathrm{~dB}(\mathrm{Z})$ lower for the posterior left upper lobe. Previously, the amplitude calibration for the tissue-borne sensitivity was obtained when the reference subject uttered a sequence of sustained vowels at normal/loud loudness, observing a level of $86.6 \mathrm{~dB}(\mathrm{Z})$, or equivalently $81.7 \mathrm{~dB}(\mathrm{~A})$, at $30 \mathrm{~cm}$ from the mouth. Therefore, it was considered that the SPL surrounding the sternal notch was approximately $85 \mathrm{~dB}(\mathrm{Z})$, for which the levels associated to air-borne sensitivities were adjusted to match this value. Consequently, broadband pink noise was presented at two intensities, $105 \mathrm{~dB}(\mathrm{Z})$ and $85 \mathrm{~dB}(\mathrm{Z})$, to allow a large SNR and match speech levels, respectively. In addition, the air-borne sensitivity of the sensors to voiced sounds was evaluated with a calibrated level of $85 \mathrm{~dB}(\mathrm{Z})$ during the protocol.

The coloring effect of the room resonances was compensated for using a prewhitening scheme. This compensation was performed using the one-third octave equalizer (Behringer DEQ2496) and a near-skin microphone as reference. Such a near-skin microphone was selected to assure that the calibration was performed as closely as possible to the sensor location. For this purpose, a Sony ECM-77B was attached to the skin using double-sided tape and a small plastic stand, in order to make it face the loudspeaker and keep a distance of 1 in from the skin. This approach was based on [1] and [29] and assures that the sound field surrounding the skin sensors have a flat response between $80 \mathrm{~Hz}$ and $8 \mathrm{kHz}$ one-third octave bands with a maximum $2 \mathrm{~dB}$ deviation between neighboring bands. This procedure was repeated for each subject to account for possible variations of the sound field due to individual body differences. The signal conditioning, digitalization, post processing, and analysis was performed as described in the previous section.

\section{Relationship Between Sensitivities}

The air-borne and tissue-borne sensitivities of biacoustic sensors are functions of the specific gains that the corresponding preamplifiers provide. In many cases, these gains are variable and defined by the user. However, the relationship between these two types of sensitivities remains constant independently of the gain. The only nonlinearity in this relationship is a result of the noise floor and distortion point of each sensor (normally referred to as the headroom). To generalize the results of this study, the concept of tissue-to-air ratio (TAR) is introduced. This relationship is considered similar to an SNR, where the "noise" 


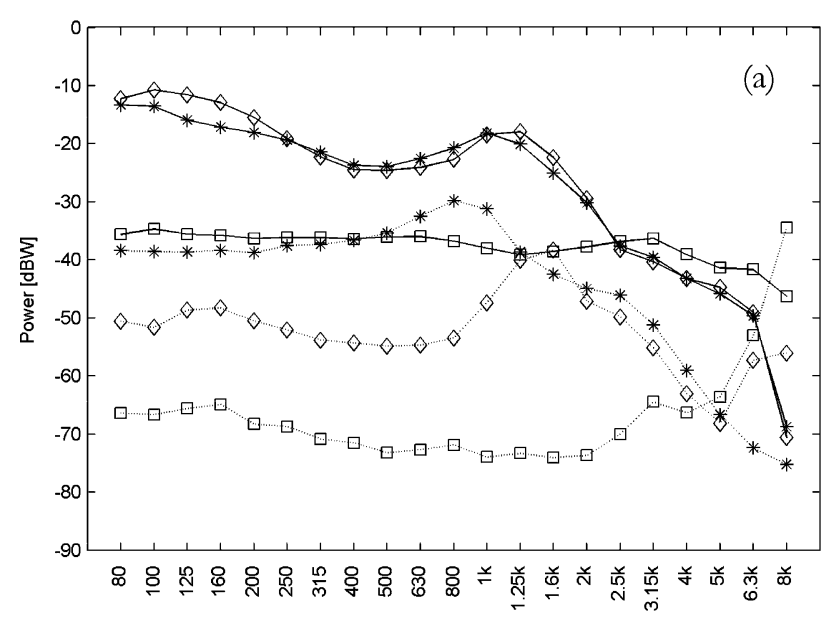

Frequency band $[\mathrm{Hz}]$

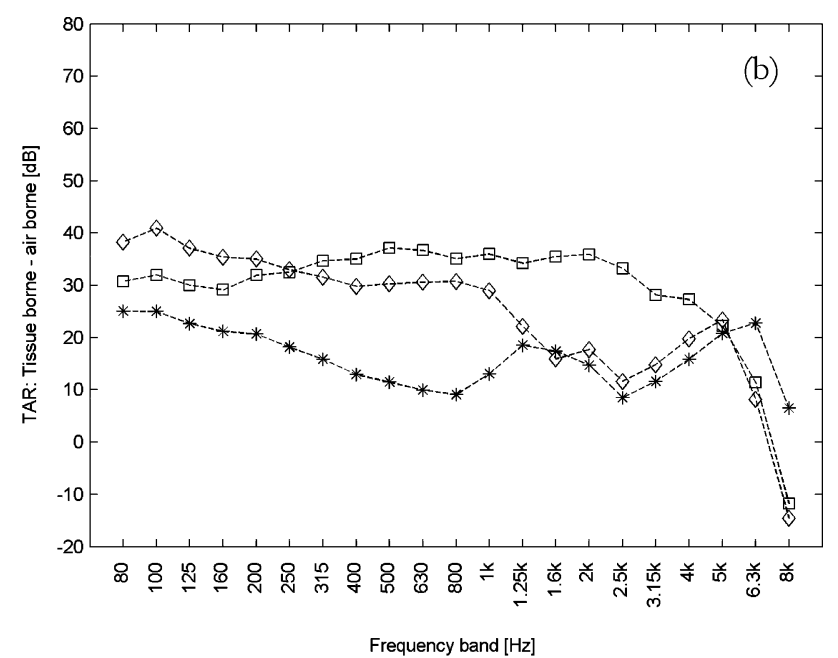

Frequency band $[\mathrm{Hz}]$

Fig. 2. Tissue-borne and air-borne sensitivities of each sensor using pink noise source. Mean air-borne sensitivity values observed on position 1 in Fig. 1 . (a) Tissue-borne and air-borne sensitivities are represented by solid lines and dotted lines, respectively. Air-coupled microphone: $*$, Siemens EMT25C: $\diamond$, Knowles BU-7135: $\square$. (b) TAR for each sensor based on the sensitivity curves. Air-coupled microphone: *, Siemens EMT25C: $\diamond$, Knowles BU-7135: $\square$.

term is associated to the air-borne component and the "signal" is associated with the tissue-borne one. The TAR can be represented as a frequency-dependent plot given by the difference (in decibels) between the tissue-borne sensitivity and the air-borne sensitivity. In this study, the TAR is primarily associated with sensitivities to voiced sounds at the sternal notch, i.e., the amplitudes of skin vibration and sound pressure were set to resemble those observed during speech at that location. The translation of these sensitivity and TAR curves for other applications (e.g., lung sounds) is discussed in Section IV.

In addition, several variables that could affect the tissue-borne and air-borne sensitivities of the sensors were investigated. These variables include the effects of subject variability, sensor location on the body, sensor attachment, passive covering of a sensor, and applied pressure on the skin surrounding the sensor. For simplicity, each passive protector or cover (e.g., earmuffs, polyvinyl chloride (PVC) caps) is referred to as a bioacoustic insulator (BAI). From an initial set of ten BAIs, three were selected via initial screening as having the most attenuation. These were two earmuffs with noise reduction ratings (NRR) 30 and NRR 33, and a 3 in diameter PVC cap. These BAIs were modified by adhering additional circumferential cushioning and acoustic filling material (Acousta-Stuf). These conditions showed the highest performance for the selected BAIs. Each BAI was evaluated using three different cover attachment forces: no force, medium force $(\sim 10 \mathrm{~N})$, and strong force $(\sim 30 \mathrm{~N})$.

\section{RESULTS}

\section{A. Tissue-Borne and Air-Borne Sensitivities}

The tissue-borne and air-borne sensitivities are presented in solid and dotted lines, respectively, for each sensor in Fig. 2(a). The results for air-borne sensitivity were obtained based on the mean values of all subjects measured at position 1 in Fig. 1. The difference between the two sensitivity curves is the TAR relation, which is presented for each sensor in Fig. 2(b). Based on these results, the Knowles accelerometer shows a higher
TAR curve, superseded only by the Siemens accelerometer for frequencies below $300 \mathrm{~Hz}$. The air-coupled microphone was shown to have the smallest TAR curve, i.e., it is easily corrupted by air-borne transmitted signals when attached to the skin. This is a result of the high sensitivity of the air-coupled microphone to both tissue-borne and air-borne sounds.

Comparisons between tissue-borne sensitivity and transverse vibration sensitivity (included in the sensor technical specifications) are possible, although deviations are expected given the differences between the testing conditions. The primary difference between the two types of sensitivities is observed at low frequencies, where the compliance of the artificial compound can alter the response of the sensor. This is more noticeable for more massive sensors, such as the Siemens accelerometer, where a stronger response below $250 \mathrm{~Hz}$ is observed in the tissue-borne sensitivity. Higher frequencies show the same essential behavior in the response of the sensor $(4 \mathrm{~dB}$ variations up to $1.25 \mathrm{kHz}$, where it shows a $-15 \mathrm{~dB}$ per octave roll-off). Variations between tissue-borne and transverse sensitivity are expected to be less significant for the Knowles accelerometer due to its relatively light weight. No comparisons of this type can be made for the air-coupled microphone, since no specifications of transverse sensitivity are available for this sensor. In addition, no comparisons with previous studies or technical datasheets can be performed for the air-borne sensitivity-dotted lines in Fig. 2(a) - of the evaluated sensors since this type of response is typically neglected or not provided.

On the other hand, previous studies [7], [8] reported the tissueborne sensitivity of the air-coupled microphone and Siemens accelerometer (same make and model). The tissue-borne sensitivities plotted as solid lines in Fig. 2(a) are in agreement with the reported data, showing only minor differences in the relative amplitudes of the peaks around 125-200 Hz and 1000$1250 \mathrm{~Hz}$. These differences are expected to be given by the different calibration schemes. Not incorporated in previous studies, the compensation for the BATT transfer function on the surface allows a flat broadband excitation on the Akton. Therefore, it 

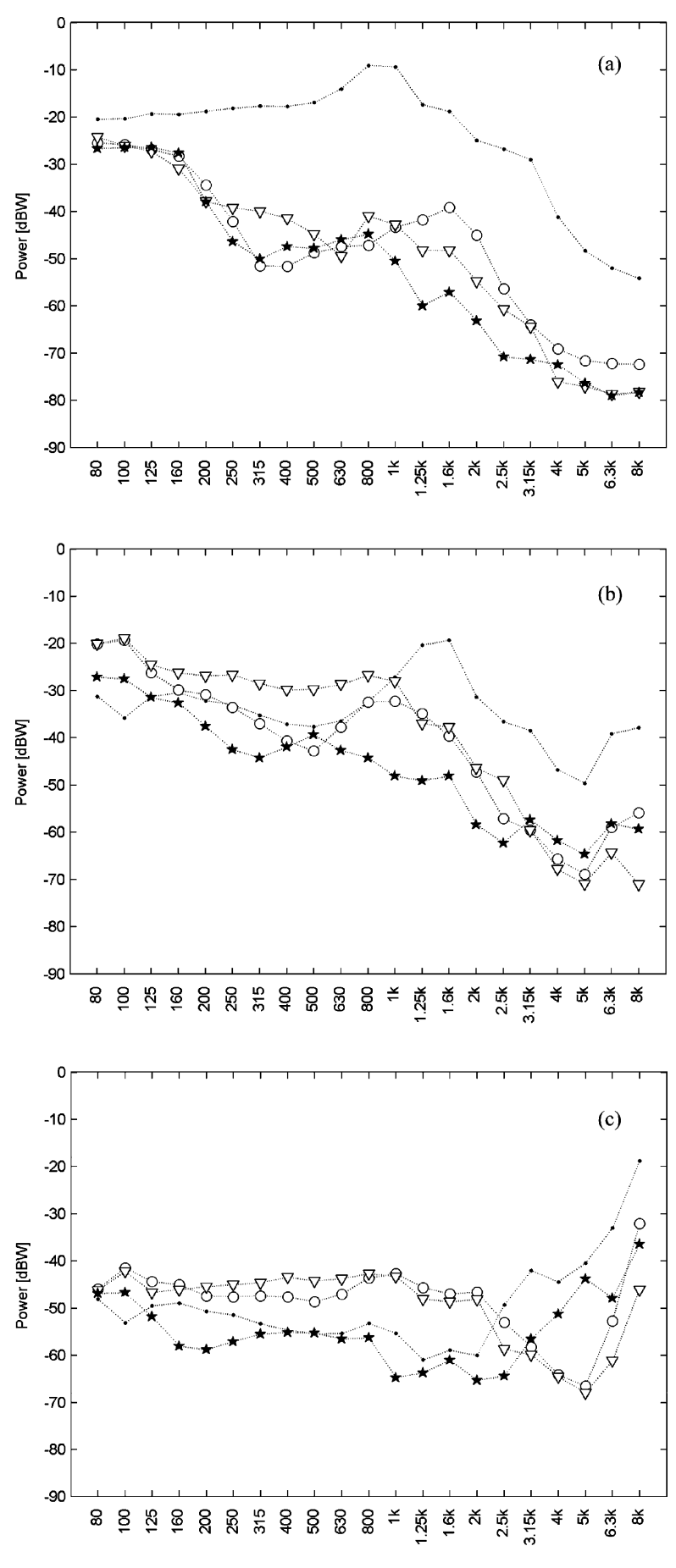

Frequency band $[\mathrm{Hz}]$

Fig. 3. Effect on the air-borne sensitivity of different BAIs applied with medium force for each sensor: (a) Air-coupled microphone. (b) Siemens EMT25C. (c) Knowles BU-7135. No protection: •, NRR30: O, NRR33: $\nabla$, PVC30: $\star$.

is believed that the current results minimize non desired effects introduced by the testing platform and better describe the tissue-borne response of the sensors.

\section{B. Acoustic Protection of Sensors with BAIs}

Results showing the air-borne sensitivity of each sensor after protecting them with the BAIs are presented in Fig. 3.

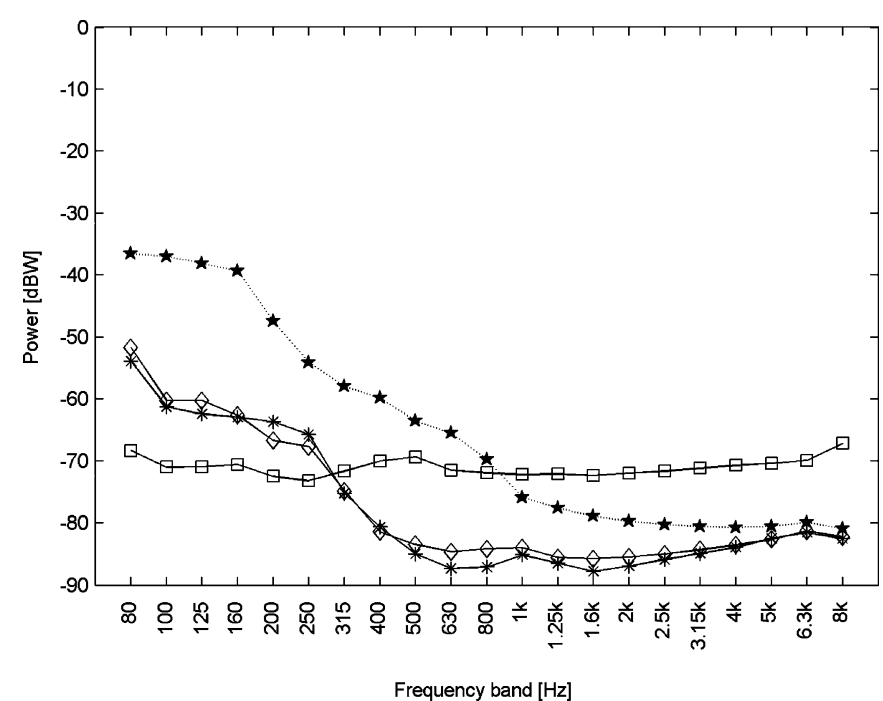

Fig. 4. Mean noise floor observed on position 1 in Fig. 1: air-coupled microphone, protected with a PVC 3 in BAI: (dotted line) $\star$, and with no BAI protection: (solid line) $*$. Siemens accelerometer with no BAI protection: (solid line) $\diamond$ and Knowles accelerometer with no BAI protection: (solid line) $\square$.

The ability of the BAIs to reduce their air-borne sensitivity depends upon each sensor. For the air-coupled microphone, the BAIs exhibit the best results since they significantly reduce its effective air-borne sensitivity. Among the three BAIs, the PVC 3 -in cap yielded the most air-borne noise protection. It should be noted that for both accelerometers, the BAIs can either reduce or amplify particular frequency components. This observation makes the BAIs generally less suitable for air-borne protection in accelerometers. However, if low-frequency amplification is desired or at least acceptable for a particular application, their usage could be justified. The presence of a BAI could potentially affect the tissue-borne sensitivity of the sensors. Tests performed in the BATT 2.0 and 2.1 showed that for accelerometers (Siemens and Knowles), the presence of the BAIs did not affect the measured spectra, whereas for the air-coupled microphone, a reduction in the sensitivity of low frequencies was observed $(-10 \mathrm{~dB}$ below $315 \mathrm{~Hz})$. This effect was proportional to the applied force. Contrasting these observations, measurements of "background body noise" on human subjects (i.e., heart sounds for the given recording conditions) were amplified at low frequencies due to the presence of the BAIs, with this effect again proportional to the applied force for all sensors. This effect is illustrated in Fig. 4, where the presence of the 3 in diameter PVC cap with medium force raised the noise floor of the air-coupled microphone, as noted in the average observed in position 1 from Fig. 1. These differences observed between BATTs and human subjects are attributed to the fact that the BATTs do not represent the changes observed in the chest wall when force is applied. In other words, the BATTs do not account for the musculoskeletal structures that will affect the transmission of body sound when pressure is applied in the skin surface.

In all, the changes introduced by the BAIs were shown to be favorable only for the air-coupled microphone. Therefore, sensitivity curves and TARs for this sensor were recomputed for the best observed conditions (PVC 3 in protected) and are presented 


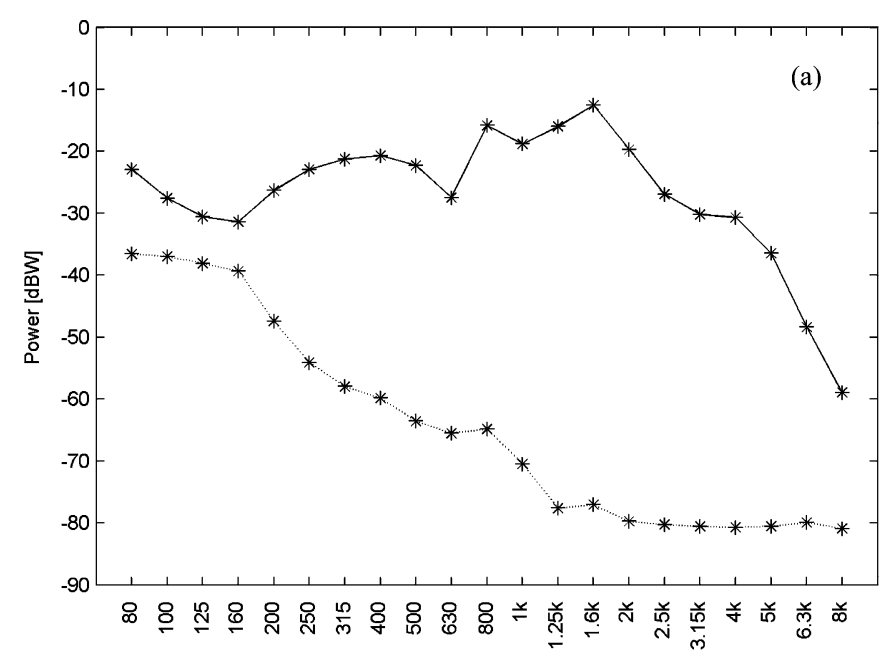

Frequency band $[\mathrm{Hz}]$

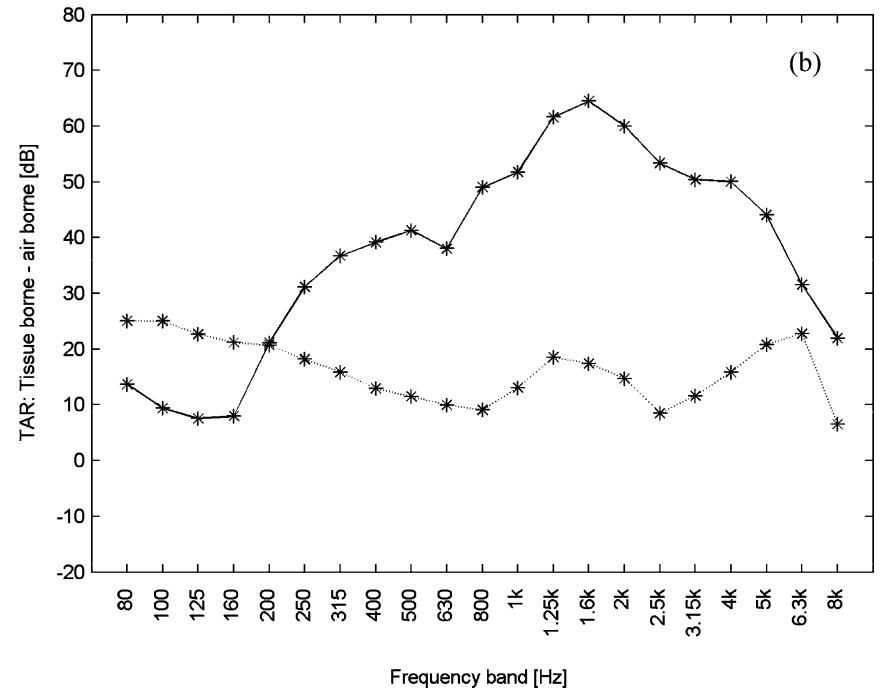

Frequency band $[\mathrm{Hz}]$

Fig. 5. Changes in the response of the air-coupled microphone due to the PVC 3 in BAI applied with medium force. (a) Tissue-borne and air-borne sensitivities measured using pink noise. Tissue-borne and air-borne sensitivities are represented by a solid line and a dotted line, respectively. (b) TAR curves. The new and original TARs are represented by a solid line and a dotted line, respectively.

in Fig. 5. Note that the increased noise floor at low frequencies hampers the TAR curves at low frequency. However, a significantly enhanced TAR curve is observed for frequencies above $315 \mathrm{~Hz}$ compared with those for the air-coupled microphone in Fig. 2.

\section{Effect of Sensor Placement and Mounting Conditions}

To evaluate the dependence of the air-borne sensitivity of the sensors upon the surface type where they are attached, such sensitivity was also measured when the sensors were placed on nontissue surfaces. This is shown for each sensor on Fig. 6, where three test surfaces were used: Akton of $1 / 4$ in, steel block of 6 in, and no surface (free field). The air-borne sensitivity for these test surfaces is contrasted with that of the chest position for convenience. It can be observed that for the air-borne sensitivity, no surface behaves similar to human skin for all sensors. This effect is attributed to the unique mounting conditions of the sensors when placed on human skin. Therefore, the tests of airborne sensitivity made on human subjects are fundamental for the purpose of this study.

The variation in sensitivity when the sensors were placed on the anterior chest (position 1 in Fig. 1), the sternal notch (position 2 in Fig. 1) and posterior RUL (position 3 in Fig. 1) was evaluated. The possible changes in the sound field between locations were also taken into account in this analysis. The differences between the body structure and tissue properties at different locations were found to have small variability $(<3 \mathrm{~dB})$ for all frequency bands of interest, except the 2.5 - and $3.15-\mathrm{kHz}$ bands, where a slightly larger deviation was observed ( $\sim 5 \mathrm{~dB})$. The main factor affecting the dispersion of the data is the subject posture, which explains the larger difference at mid-high frequencies. In all, this response illustrates that differences in skin mounting conditions, such as subcutaneous fat thickness, have only a minor effect on the air-borne sensitivity of the sensors. The fact that the air-borne sensitivity remains relatively constant for different body location is convenient for future studies since the observations made for each sensor in this study can be extrapolated to different body locations.

The variability between subjects was also evaluated. Factors that primarily affect the dispersion of the data are subject posture and body configuration. The air-borne sensitivity across subjects showed deviations lesser than $3 \mathrm{~dB}$ for all the frequency bands. Thus, the general air-borne sensitivity trends of the sensors were observed to be consistent across subjects.

\section{APPLICATION NOTES}

\section{A. Using Sensitivities to Discriminate Components}

In order to illustrate how the air-borne and tissue-borne sensitivities can be used to discriminate if the signal detected by a bioacoustic sensor is primarily a product of tissue- or air-borne components, the following example was considered. A recording of voiced sounds on the skin surface (sustained vowel /a/) over the anterior right lobe of the lung was performed using the air-coupled microphone protected with the 3 in PVC cap with a medium force holding it against the skin. The one-third octave analysis of this recorded signal is presented in Fig. 7 along with the air-borne sensitivity of the sensor for this condition. It can be observed from this figure that for frequencies below $1.6 \mathrm{kHz}$, the signal is at least $10 \mathrm{~dB}$ stronger than the air-borne sensitivity. This implies that the sensor was primarily detecting tissue-borne sounds in that range. To further investigate this phenomenon, an estimate of the expected tissue-borne component is included in Fig. 7. This estimation was performed using the calibrated BATT excited with a sustained vowel, reducing the amplitude of vibration to mimic chest vibration instead of that of the sternal notch. The resulting estimated signal is sufficiently close to the actual measured signal up to $1.6 \mathrm{kHz}$, where the effect of the noise floor becomes significant. This illustrates that the 

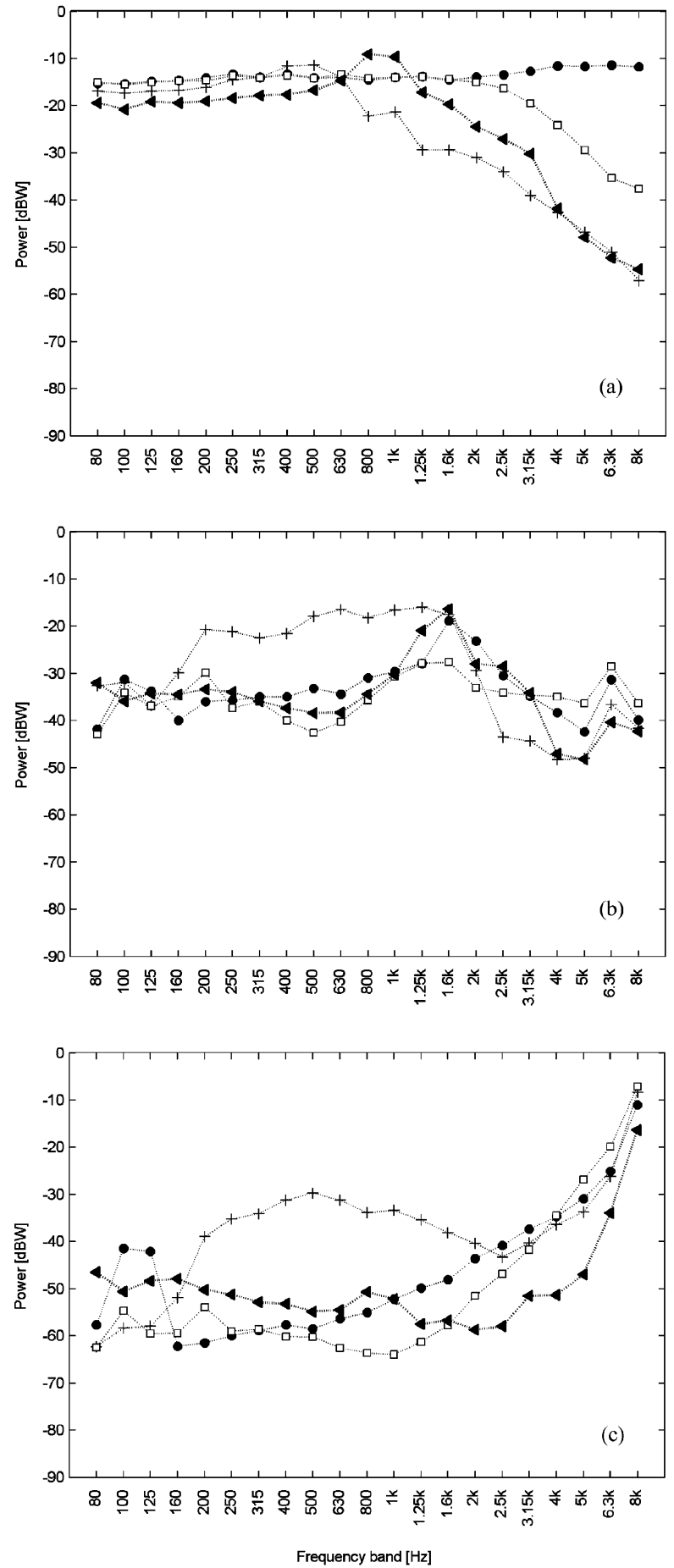

Fig. 6. Effect of different surfaces on the air-borne sensitivity. (a) Air-coupled microphone. (b) Siemens EMT25C. (c) Knowles BU-7135. Akton: +, steel: $\square$, posterior chest (RUL): 4, free field:

air-borne sensitivity curve would represent a threshold that allows the discrimination of components. Any response of the sensor above this curve would be largely due to tissue-borne components. The tissue-borne sensitivity of the sensor could be used as shown a priori to create estimates of tissue-borne components that can be contrasted with the actual measured signal.

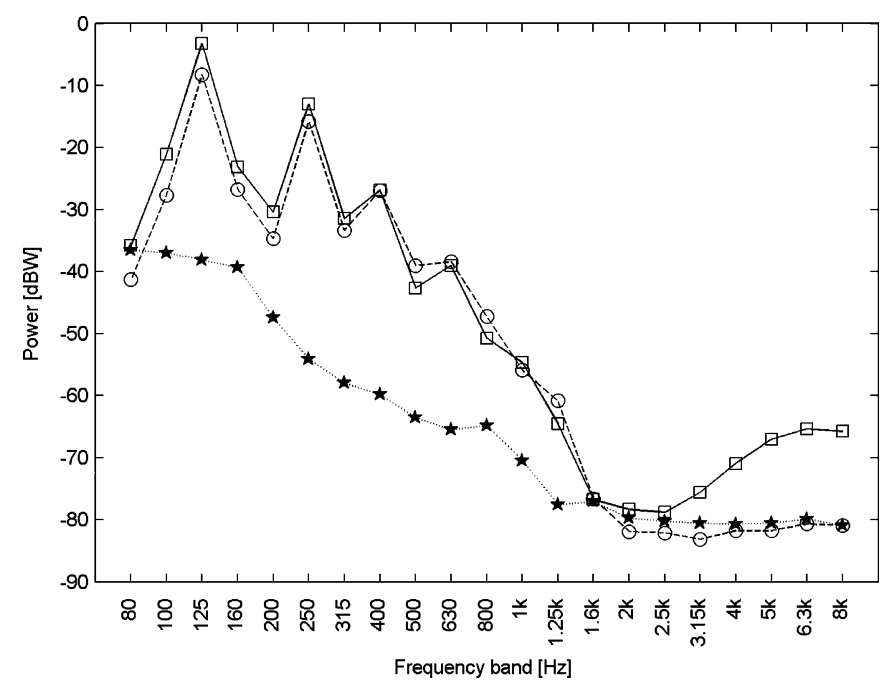

Fig. 7. Application of air-borne and tissue-borne sensitivities during the discrimination between components. Sensor: air-coupled microphone protected with PVC $3^{\prime \prime}$ on the chest. Measured signal during vowel /a/: $\square$, estimated signal for vowel /a/ for tissue-borne components only: $\bigcirc$, air-borne sensitivity for such condition: $\star$.

\section{B. Translating Sensitivities and TAR Curves for Other Applications}

The example presented in the previous section introduces the idea of translation of the sensitivity curves. The sensitivity curves from Figs. 2(a) and 5(a) could be translated (i.e., shifted up or down by a certain offset), as long as the overlapping effect of noise floor that bounds the translation is considered. The noise floor that needs to be considered for each sensor is presented in Fig. 4. In order to achieve this translation, it is necessary to know the offset in amplitude of vibration and sound level pressure between the conditions presented in this study and those of interest to the reader. This requires comparing both the amplitude of vibration between the sternal notch and the new location and condition of interest. At the same time, it is necessary to compare the SPL surrounding the sternal notchestimated at $85 \mathrm{~dB}(\mathrm{Z})$ - with that surrounding the new location and condition of interest. These comparisons will yield the offset needed to translate both sensitivities curves, and a new TAR curve could be computed using these curves. Alternatively, it is possible to repeat the set of procedures performed in this study, adjusting the levels of amplitude for air-borne and tissue-borne excitation to describe a different desired condition.

\section{CONCLUSION}

The results of this study indicate that air-borne components can clearly affect skin surface measurements of body sounds and that the selection and understanding of the sensor behavior for the given recording conditions becomes critical. Air-borne corruption of skin surface measurements of voiced sounds was observed to be substantial. The proposed sensitivity and TAR curves aid the selection of bioacoustic sensors for different audible acoustic applications involving skin surface measurements. However, the construction of these curves depends on the 
desired application. Although the sensitivities presented in this study are based on skin radiation of voiced sounds at the sternal notch, the proposed methods could serve as a guide for other applications. The gain of a bioacoustic sensor plays a significant role, compromising the absolute tissue-borne and air-borne sensitivities, but does not affect the TAR. The headroom (noise floor versus distortion point) is the limiting factor of the linearity of this relation. The selection of the sensors is also highly related to the application. Based on the sensitivity and TAR curves with no BAI protection, the best sensor appears to be the Knowles accelerometer. However, this sensor is less sensitive to tissue-borne sounds, for which it is better suited to relatively high-amplitude vibration cases (e.g., recordings of voiced sounds at the sternal notch). The TAR curve for the air-coupled microphone is significantly enhanced using BAIs, particularly at mid-high frequencies. However, handling the air-coupled sensor along with a BAI can be more cumbersome than the other sensors and it is not suitable for all body locations. For lowfrequency applications (below $200 \mathrm{~Hz}$ ), the best performance was achieved by the Siemens accelerometer.

\section{REFERENCES}

[1] Methods for the Calibration of Vibration and Shock Pick-Ups. Part 15: Testing of Acoustic Sensitivity (Méthodes pour l'étalonnage de capteurs de vibrations et de chocs. Partie 15, Essai De Sensibilité Acoustique), 1st ed. Geneva, Switzerland, International Organization for Standardization, ISO 5347-15-1993, 1993.

[2] Methods for the Calibration of Vibration and Shock Pick-Ups. Part 11: Testing of Transverse Vibration Sensitivity (Méthodes pour l'étalonnage de capteurs de vibrations et de chocs. Partie 11, Essai De Sensibilité Aux Vibration Transversales), 1st ed. Geneva, Switzerland, International Organization for Standardization, ISO 5347-11-1993, 1993.

[3] G. R. Wodicka, S. S. Kraman, G. M. Zenk, and H. Pasterkamp, "Measurement of respiratory acoustic signals. Effect of microphone air cavity depth," Chest, vol. 106, pp. 1140-1144, Oct. 1994.

[4] S. S. Kraman, G. R. Wodicka, Y. Oh, and H. Pasterkamp, "Measurement of respiratory acoustic signals. Effect of microphone air cavity width, shape, and venting," Chest, vol. 108, pp. 1004-1008, Oct. 1995.

[5] H. Pasterkamp, S. S. Kraman, P. D. DeFrain, and G. R. Wodicka, "Measurement of respiratory acoustical signals. Comparison of sensors," Chest, vol. 104, pp. 1518-1525, Nov. 1993.

[6] H. Pasterkamp, S. S. Kraman, and G. R. Wodicka, "Respiratory sounds. Advances beyond the stethoscope," Amer. J. Respir. Crit. Care Med., vol. 156, pp. 974-987, Sep. 1997.

[7] S. S. Kraman, G. A. Pressler, H. Pasterkamp, and G. R. Wodicka, "Design, construction, and evaluation of a bioacoustic transducer testing (BATT) system for respiratory sounds," IEEE Trans. Biomed. Eng., vol. 53, no. 8, pp. 1711-1715, Aug. 2006.

[8] S. S. Kraman, G. R. Wodicka, G. A. Pressler, and H. Pasterkamp, "Comparison of lung sound transducers using a bioacoustic transducer testing system," J. Appl. Physiol., vol. 101, pp. 469-476, Aug. 2006.

[9] H. Pasterkamp, G. R. Wodicka, and S. S. Kraman, "Effect of ambient respiratory noise on the measurement of lung sounds," Med. Biol. Eng. Comput., vol. 37, pp. 461-465, Jul. 1999.

[10] S. B. Patel, T. F. Callahan, M. G. Callahan, J. T. Jones, G. P. Graber, K. S. Foster, K. Glifort, and G. R. Wodicka, "An adaptive noise reduction stethoscope for auscultation in high noise environments," J. Acoust. Soc. Amer, vol. 103, pp. 2483-2491, May 1998.

[11] R. T. H. Laennec, De l'Auscultation Médiate, ou, Traité du Diagnostic des Maladies des Poumons et du Coeur: Fondé principalement sur ce Nouveau Moyen d'Exploration, J.-A. Brosson, et J.-S. Chaudé, 1819.

[12] A. Cohen and A. D. Berstein, "Acoustic transmission of the respiratory system using speech stimulation," IEEE Trans. Biomed. Eng., vol. 38, no. 2, pp. 126-132, Feb. 1991.

[13] J. D. Sapira, “About egophony,” Chest, vol. 108, pp. 865-867, Sep. 1995.
[14] J. Xu, J. Cheng, and Y. Wu, "A cepstral method for analysis of acoustic transmission characteristics of respiratory system," IEEE Trans. Biomed. Eng., vol. 45, no. 5, pp. 660-664, May 1998.

[15] F. L. Jones, Jr., "Poor breath sounds with good voice sounds. A sign of bronchial stenosis," Chest, vol. 93, pp. 312-313, Feb. 1988.

[16] R. E. Hillman, J. T. Heaton, A. Masaki, S. M. Zeitels, and H. A. Cheyne, "Ambulatory monitoring of disordered voices," Ann. Otol. Rhinol. Laryngol., vol. 115, pp. 795-801, Nov. 2006.

[17] H. A. Cheyne, H. M. Hanson, R. P. Genereux, K. N. Stevens, and R. E. Hillman, "Development and testing of a portable vocal accumulator," J. Speech Lang. Hear. Res., vol. 46, pp. 1457-1467, Dec. 2003.

[18] J. G. Svec, P. S. Popolo, and I. R. Titze, "Measurement of vocal doses in speech: Experimental procedure and signal processing," Logoped. Phoniatr. Vocol., vol. 28, pp. 181-192, 2003.

[19] J. Sundberg, "Chest wall vibrations in singers," J. Speech Hear. Res., vol. 26, pp. 329-340, Sep. 1983.

[20] J. G. Svec, I. R. Titze, and P. S. Popolo, "Estimation of sound pressure levels of voiced speech from skin vibration of the neck," J. Acoust. Soc. Amer, vol. 117, pp. 1386-1394, Mar. 2005.

[21] K. N. Stevens, D. N. Kalikow, and T. R. Willemain, "A miniature accelerometer for detecting glottal waveforms and nasalization," J. Speech Hear. Res., vol. 18, pp. 594-599, Sep. 1975.

[22] R. P. Lippmann, "Detecting nasalization using a low-cost miniature accelerometer," J. Speech Hear. Res., vol. 24, pp. 314-317, Sep. 1981.

[23] J. Moon, "The influence of nasal patency on accelerometric transduction of nasal bone vibration," Cleft Palate J., vol. 27, pp. 266-270, Jul. 1990, (discussion 270-274).

[24] W. E. Cayley, Jr., "Diagnosing the cause of chest pain," Amer. Family Physician, vol. 72, pp. 2012-2021, Nov. 2005.

[25] M. A. Spiteri, D. G. Cook, and S. W. Clarke, "Reliability of eliciting physical signs in examination of the chest," Lancet, vol. 1, pp. 873-875, Apr. 16, 1988.

[26] J. A. Fiz, J. Gnitecki, S. S. Kraman, G. R. Wodicka, and H. Pasterkamp, "Effect of body position on lung sounds in healthy young men," Chest, vol. 133, pp. 729-736, Mar. 2008.

[27] Specification for Octave-Band and Fractional-Octave-Band Analog and Digital Filters, Acoustical Society of America and American National Standards Institute, ANSI S1.11-2004, 1986.

[28] Criteria for Evaluating Room Noise, Acoustical Society of America and American National Standards Institute, ANSI S12.2-1995 (R1999), 1995.

[29] Microphone-In-Real-Ear and Acoustic Test Fixture Methods for the Measurement of Insertion Loss of Circumaural Hearing Protection Devices, Acoustical Society of America and American National Standards Institute, ANSI S12.42-1995 (R2004), 1995.

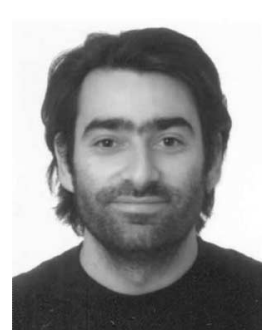

Matías Zañartu (S'08) received the B.S. and professional (C.E.) degrees in acoustical engineering from Universidad Tecnológica Vicente Pérez Rosales, Santiago, Chile, in 1996 and 1999, respectively, and the M.S. degree in electrical and computer engineering in 2006 from Purdue University, West Lafayette, IN, where he is currently working toward the Ph.D. degree in electrical and computer engineering.

Since 2006, he has been a Research Assistant at Purdue University. During 2008, he was a Visiting Researcher at the Center for Laryngeal Surgery and Voice Rehabilitation, Massachusetts General Hospital, Boston. His current research interests include biomedical acoustics, speech production, acoustic phonetics, and auditory and speech signal processing.

Mr. Zañartu was the recipient of a Fulbright Scholarship, an Institute of International Education (IIE)-Barsa Scholarship, and a Qualcomm Q Award of Excellence. He is a member of the Acoustical Society of America, the Biomedical Engineering Society, the Society of Hispanic Professional Engineers, and the Acoustical Society of Chile. 


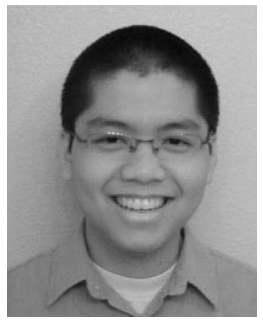

Julio C. Ho (S'08) was born in Panama City, Panama, in 1984. He received the B.S. degree (with distinction) in electrical and computer engineering, in 2006, from Purdue University, West Lafayette, IN, where he is currently working toward the Ph.D. degree in biomedical engineering.

His current research interests include biomedical acoustics, biomedical instrumentation, and signal processing.

Mr. Ho is a member of Tau Beta Pi and Etta Kappa $\mathrm{Nu}$. He was the recipient of the A. F. Welch Memorial Scholarship in 2003 and 2004, the Kimberly-Clark Industrial Affiliates Scholarship in 2005, and the Schlumberger Industrial Affiliates Scholarship in 2006.

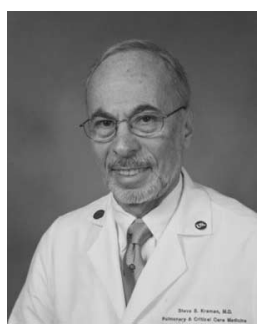

Steve S. Kraman (M'82-SM'07) was born in Chicago, IL, in 1944. He received the B.S. and M.D. degrees from the University of Puerto Rico, Río Piedras, in 1968 and 1973, respectively.

In 1978, he was engaged in Postgraduate training in internal medicine and then pulmonary and critical care medicine in New York. He is currently a Professor of internal medicine at the University of Kentucky, Lexington. He has performed research in various aspects of respiratory acoustics that has been published in a variety of medical and engineering journals.

Prof. Kraman is a Fellow of the American College of Chest Physicians and is a member of the American Thoracic Society.

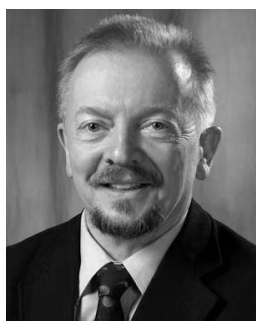

Hans Pasterkamp received the M.D. degree from Medical University Lübeck, Lübeck, Germany, in 1976.

He was engaged in training in pediatrics at the Medical University Lubeck from 1976 to 1981, and in pediatric respirology in the Faculty of Medicine, University of Manitoba, Winnipeg, from 1981 to 1984, where he has been a Full Professor and the Head of the Section of Respirology, Department of Pediatrics and Child Health since 1997. His current research interests include respiratory sounds and their relation

to pulmonary mechanics.

Prof. Pasterkamp is a member of the American Thoracic Society, the European Respiratory Society, the Society for Pediatric Research, the Canadian Pediatric Society, the Acoustical Society of America, and the American Academy of Sleep Medicine. Since 1986, he has been a Fellow of the Royal College of Physicians and Surgeons of Canada, Ottawa, ON, Canada. Since 2003, He has been the Deputy Editor of the journal Pediatric Pulmonology.

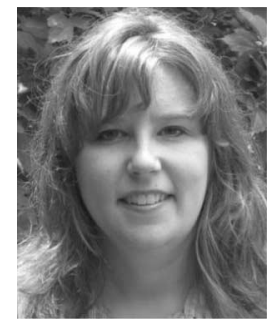

Jessica E. Huber received the B.A. degree in english from St. John Fisher College, Rochester, NY, in 1992, and the M.S. degree in speech-language pathology and the Ph.D. degree in speech science from the University at Buffalo, Buffalo, NY, in 1997 and 2001, respectively.

She is currently an Associate Professor in the Department of Speech, Language, and Hearing Sciences, Purdue University, West Lafayette, IN. Her current research interests include the physiology of speech production, the effects of Parkinson's disease and typical aging on speech and language, and the interaction between speech, language, and cognition.

Prof. Huber is a member of the American Physiological Society, the American Speech-Language-Hearing Association, and the Acoustical Society of America. She holds a clinical certification as a Speech-Language Pathologist.

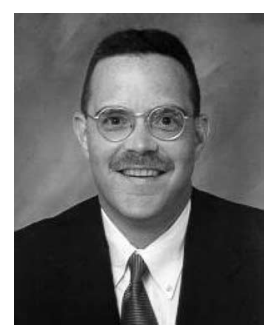

George R. Wodicka (S'81-M'82-SM'93-F'02) received the B.E.S. degree in biomedical engineering from Johns Hopkins University, Baltimore, MD, in 1982 , and the S.M. degree in electrical engineering and computer science and the Ph.D. degree in medical engineering from Massachusetts Institute of Technology, Cambridge, MA, in 1985 and 1989, respectively.

He is currently a Professor and the Head of the Weldon School of Biomedical Engineering and a Professor of electrical and computer engineering at Purdue University, West Lafayette, IN. His current research interests include biomedical acoustics, acoustical modeling, and signal processing.

Prof. Wodicka was the recipient of the National Science Foundation Young Investigator Award. He is a Fellow of the American Institute for Medical and Biological Engineering and a Guggenheim Fellow. 